\title{
A Reappraisal of Online Mathematics Teaching Using LaTeX
}

\author{
doi:10.3991/ijet.v4i4.970 \\ E. Costello, S. Fox and E. Walsh \\ Dublin City University, Dublin, Ireland
}

\begin{abstract}
The mathematics language LaTeX is often seen outside of academic circles as a legacy technology that is awkward to use. MathML - a verbose language designed for data-exchange, and to be written and understood by machines - is sometimes by contrast seen as something that will aid online mathematics and lack of browser support for it bemoaned. However LaTeX can already do many of the things that MathML might promise. LaTeX is here proposed as a language from which small fragments, with concise syntax, can be used by people to easily create and share mathematical expressions online. The capability to embed fragments of LaTeX code in online discussions is described here and its impact on a group of educators and learners evaluated. Here LaTeX is posited as a useful tool for facilitating asynchronous, online, collaborative learning of mathematics.
\end{abstract}

Index Terms-online learning, LaTeX, mathematics teaching

\section{INTRODUCTION}

Pencil and paper and chalk and blackboard have proved powerfully enduring and pervasive technologies in the teaching of Mathematics. Finding equivalent tools for the online classroom has preoccupied researchers for a long time and no real de facto standard approach has hitherto emerged. The wide variety of forms through which mathematical concepts can be expressed is partly to blame e.g. a tool for graphing may not be a suitable one for algebra. A huge semantic space is also apparent, where for example the same symbol or notation can be used to represent different concepts in different contexts. Another problem is that there are so many forms which we generally consider as conventions but computers must consider as rules (e.g. $1 x=x$ or $2 x=x 2$ ). After we consider these problems, and investigate different ways of representing mathematics accurately in digital form, there then arises the task of evaluating how feasible these approaches are for education and how practically useful and effective they are for teachers and learners.

Here we present our research into a small subsection of this problem. We focus on an approach to mentoring and tutoring students online in mathematics as part of an undergraduate degree in Information Technology for distance learners. We describe how this approach evolved from a simple experiment and how we evaluated it over several student cohorts.

\section{A. Delivering an IT Programme Online}

Oscail provides distance education to students of Dublin City University (DCU) in Ireland. Oscail's BSc. in
Information Technology is a programme consisting of 16 modules. Students study part time by distance and can exit with either a diploma or an honours degree. The programme has migrated from a traditional paper-based correspondence model to one where almost everything is now online. For an overview of this transition see [1].

During the IT programme's phased online transition all of the instructional materials (course notes, schedules, assignments, remedial and other ancillary materials) for each module were digitized and provided to students through the Virtual Learning Environment (VLE) Moodle. One of the reasons for moving online was that attendances at face-to-face tutorials were decreasing. Tutorials were not mandatory but attendance showed correlation with performance and persistence. In an effort to provide more flexible teaching support tutorials were offered online in addition to face-to-face.

It was possible to develop engaging forms of assessment structured around forum discussions in Moodle, and this work was concentrated on subjects that were discursive in nature rather than more fact and theory driven ones [2]. These assessments revolved around collaborative, peer-tutored discussions which were moderated and marked by online tutors [3]. This asynchronous, online, collaborative learning model is based on wider research on reflective learning [4] [5].

\section{B. Migrating Mathematics Online}

There were four modules of the programme which, due to their Mathematical content, were only partially moved online. The students were given access to the online course materials and resources in these modules. They also had access to discussion forums where they could discuss the course content and the assignments with each other and an online tutor. However they still submitted their assignments by terrestrial post (as opposed to emailing or uploading a file). Many academic stakeholders were sceptical of moving away from handwritten mathematics assignments, believing any form of typed mathematics assignment would be an unacceptable burden for both students and assignment markers. Like electronic assignment submission, many academic stakeholders were also wary of online mathematics tutoring. The rationale for online tutoring was already weakened by the fact that assignments would not be submitted online. Thus we decided to investigate the feasibility and the value of online mathematics learning through Moodle before mandating electronic assignment submission. 


\section{Online Resources}

The students in question form an unusual cohort in the Irish context in that there is no entry requirement for secondary school mathematics attainment, provided they are over 23. For certain mathematics and statistics modules however, Ordinary Level Mathematics in the Irish Leaving Certificate is advised (and these modules are mandatory). Thus one of the first online supports we gave to students was the provision of remedial material. This material covered topics such as manipulation of fractions; basic algebra and introductory trigonometry, a knowledge of which is assumed in the course text proper.

Banks of multiple choice and text entry questions were both developed internally and repurposed from external sources. These questions were self-diagnostic in nature, directing the students to the relevant support materials based on their performance. Richer interactive flash applets were also developed. These involved step by step problem solving, or explored concepts such as solving linear systems using a matrix algebra calculator. This particular resource proved most popular with students. Usage levels were sporadic, mostly clustered around exam times or during a short period when it was announced the resources were available [6].

The latest addition we have made has been the development of online video. This was done using screen recording software (Camtasia Studio) and a tablet PC. It has proved a very cost effective method of producing high quality learning resources quickly and the student feedback has been overwhelmingly positive.

\section{MATHEMATICS DiscuSSIONS ONLINE}

All the efforts described so far to enrich the online environment for students do not involve human to human interaction. As such they exist in a vacuum. The basic educational ingredient of communication is missing. Representing mathematical concepts online is not an insurmountable problem. It becomes harder when we want to conduct some form of dialogue. Levanthall [7] carried out in-depth investigations into the exact information conveyed in social interactions when teaching mathematics. She analysed video footage of classroom scenarios and derived specific criteria for an idealised online mathematics learning environment which are paraphrased below:

1. Ability to share and annotate another user's document in real time.

2. A way of displaying multiple documents and highlighting sections, then drawing links to a synchronous discussion

3. Some method of pointing to and using emoticons for pointing words - 'this', 'that' etc.

4. A way of reading an equation aloud so that a student can hear the words that go with the symbols.

5. A quick sketch tool with drag and drop elements for frequently used items like axes.

6. A reusable, re-editable equation in a discussion thread so that elements of a previous post can be quickly cut then edited and posted in the current message to save time.

7. Some way of showing 3-D gestures to indicate position, direction etc. associated with diagrams.
Smith and Ferguson [8] created a similar list of criteria, arriving at their finding through email interviews with 40 distance mathematics tutors. They summarized:

[The tutors] wanted to be able to put mathematical notation directly into threaded discussions and online postings and to be able to do quick conceptual sketches, have access to a graphing calculator and an equation solver, and to be able to import files from common math software such as Excel, Mathcad, Mathematica, Maple, etc.

Their fully elaborated requirements are shown below:

1. Communicate with diagrams with naturalness.

2. Communicate with formulas and text with fluidity and naturalness.

3. Simple and easy to use.

4. Seamlessly integrate diagrams, formulas/math notation and text.

5. Communicate two-way (with diagrams, formulas and text) between instructor-students, instructorstudent, student-instructor and student-student in public and private one-to-one modalities.

6. Archive all postings of a course in an organized way.

7. Postings are 'copy able' and 'modifiable'.

8. Asynchronous systems as well as synchronous modalities.

9. Postings syntactically retain the semantic mathematical meaning of expressions.

10. Integrates with the online distance education environment.

11. The System is robust.

Both of these efforts [7] [8] are concentrated on an idealized, generalizable system. They share features, such as the very specific ability to copy and modify postings, and the more general ideas of simplicity and the seamless integration of tools with the learning environment

\section{LATEX AND ONLINE TUTORING}

LaTeX (pronounced 'Lay Tech') is a typesetting language which has grown out of an earlier system called TEX. (LaTeX is itself now superseded by LaTeX 2e.). LaTeX refers to a larger superset of TEX which includes additional functionality (macros) for detailed typesetting of documents for publication, however here we will use the term LaTeX mostly to refer to the parts of the language directly concerned with representing mathematics. LaTeX is often seen as a "legacy technology" [9] that is awkward to use [10] and commentators are often dismissive of its capabilities (such as comparing it to raster image formats) [11]. Other researchers do not even consider LaTeX for teaching, but have instead been attracted by promises made by MathML and may conflate implementations of MathML with the standard itself somewhat [8] or be unaware of the possible advantages of LaTeX over MathML in this context.

One of the main reasons for the original TEX was to provide a way for mathematics to be easily typed so that the typed code could then automatically be rendered into traditional notation by a software program. It was this feature that we aimed to use. Fragments of LaTeX markup can be embedded in discussion forum posting in Moodle. The user encloses the parts of text they wish to be 
rendered within two sets of dollar signs. When they submit their posting, the Moodle server parses the input, finds any LaTeX fragments and generates a GIF image showing the expression in typeset form. Users who read postings containing LaTeX fragments will see mathematical expressions appear seamlessly within surrounding text. Crucially, by clicking on the mathematical expression they are given access to the LaTeX source code. By copying, they can then use this code to create posts of their own. In this way, the effort of modifying a pre-existing mathematical expression is much less than creating one from scratch.

There were several key advantages that were apparent straight off to using LaTeX in Moodle discussions:

- No plugins are needed by users.

- LaTeX is a core Moodle module so it should work straight out of the box on most Moodle configurations.

- The postings are 'copyable and modifiable' - a key criterion for both the frameworks of [7] and [8].

- The LaTeX function is native to Moodle and thus seamlessly integrated.

- It is very easy to learn the basic mechanism (leaving aside the LaTeX syntax).

- It is relatively easy to learn and use basic LaTeX syntax.

In contrast to the researchers cited above our aims were modest. Firstly we did not attempt to find an allencompassing solution but rather one that was 'good enough' to discuss specific mathematical problems in the specific context of our mathematical modules. An analysis was made of the module syllabi for which we wished to use online tutoring. For each module all mathematical terms used were identified and a guide was written which gave the LaTeX code for each of these terms. A summary of how many LaTeX forms and idioms were identified is given in Table 1 (which also gives an outline of the syllabus). These were given in electronic format so they were capable of being copied and modified. The online tutor was given very brief training (almost none was necessary). The students were required to typeset some simple expressions in LaTeX as part of their first assignment. After this the use of LaTeX was entirely optional.

TABLE I.

\begin{tabular}{|l|c|}
\hline \multicolumn{1}{|c|}{ Topic } & $\begin{array}{c}\text { Different symbols or } \\
\text { conventions identified }\end{array}$ \\
\hline Basic Algebra & 30 \\
\hline Linear Algebra & 7 \\
\hline Trigonometry & 13 \\
\hline Differential Calculus & 9 \\
\hline Integral Calculus & 2 \\
\hline Probability & 3 \\
\hline Probability Distributions & 3 \\
\hline Statistical Estimation & 3 \\
\hline Testing Hypotheses & 0 \\
\hline Chi-Square Distribution & 1 \\
\hline Correlation & 2 \\
\hline Linear Regression & 2 \\
\hline
\end{tabular}

\section{EVALUATION}

Many researchers emphasise the danger that learning to use a technology can swamp the learning of the topic at hand. McArthur et al. [12] sum this up well: "In any rich inquiry, how can you arrange for students to focus on the important skills when relatively unimportant ones must also be applied and may require much of the cognitive effort? This is a long-standing dilemma in pedagogy."

That using LaTeX is too difficult for staff and students to learn and use is a common preconception. Many research efforts are concentrated on drag and drop equation builders, implying that typing is mathematics is unfeasible. This was also the reaction of tutors at the outset of this project to the introduction of LaTeX. In initial interviews they felt that using LaTeX would be time consuming. However in evaluation surveys of students in modules that went on to use LaTeX the results were not negative. Table 2 gives the outcomes of survey evaluation of one particular module, Management Sciences 1, which used LaTeX for online discussion. On average (over three years) there were 946 postings per year in the course with 395 of those posts containing LaTeX (about 40\%).

The results over three years show that students (albeit by a small margin) found LaTeX useful, easy to use, and enjoyable. On a likert scale - where 1 is strongly agree, and 5 is strongly disagree, the following averaged scores were obtained:

TABLE II.

\begin{tabular}{|c|c|c|c|c|c|c|}
\hline Year & \multicolumn{3}{|c|}{ LaTeX in Moodle } & \multicolumn{2}{c|}{ Students } \\
\hline & Useful & $\begin{array}{c}\text { Easy to } \\
\text { use }\end{array}$ & $\begin{array}{c}\text { Enjoyable } \\
\text { to use }\end{array}$ & $\begin{array}{c}\text { Used } \\
\text { often }\end{array}$ & $\begin{array}{c}\text { Response } \\
\text { rate }\end{array}$ & $n$ \\
\hline 2008 & 2.3 & 2.6 & 2.7 & $77 \%$ & $50 \%$ & 42 \\
\hline 2007 & 2.2 & 3.5 & 3.1 & $64 \%$ & $45 \%$ & 38 \\
\hline 2006 & 2.8 & 2.7 & 2.7 & $80 \%$ & $46 \%$ & 53 \\
\hline Total & 2.43 & 2.93 & 2.83 & $74 \%$ & $47 \%$ & 133 \\
\hline
\end{tabular}

One thing that is interesting is that respondents in all groups were in agreement that using LaTeX in Moodle is useful even though it might not be so enjoyable or easy to use. Even though the scores for ease of use and enjoyableness are not convincing, they still go against some conventional wisdom which holds that LaTeX is unworkable and not feasible for students. The more favourable scores for usefulness in the second year (and in the average of the three groups taken together - 2.36) versus ease of use show that students are discerning evaluators who do not necessarily equate higher workloads with lower overall quality of education [13].

\section{SyntaX OF ONLINE Discussions}

When LaTeX input to discussions is parsed by Moodle it tries to render the expression and then output the results as best it can. It has no Computer Algebra (CA) engine and so does not understand semantics of expressions. It cannot manipulate expressions into a standard form, reduce or expand them nor test for their equivalence. Neither is there any direct grade incentive for students to be rigorous in their use of LaTeX. Students tended to use LaTeX sometimes and sometimes not. Sometimes they mixed bits of LaTeX with free text. Many conventions common from computing, such as the use of the asterisk 
for the multiplication operator and the forward slash for division, were used. Often students mixed LaTeX fragments with these other forms for instance showing nested fractions is difficult without using classical notation. A computer programming language might use sets of parentheses to separate terms in a nested fraction however this is not easy to read and comprehend. In cases like this students took a pragmatic approach. For example in one discussion, concerning a nested fraction, two students used the long LaTeX form for the outer fraction, that is they used the macro command Irac followed by two sets of opening and closing curly braces enclosing the numerator and denominator i.e. Ifac\{numerator\}\{denominator\}. For the inner, nested fraction the students simply used a forward slash between numerator and denominator. This fraction was itself a power of another number i.e. $9^{\wedge}\{3 / 2\}$ (the caret character is used to indicate a superscript term). The code and how it was rendered is shown in Fig. 1.

$$
\left\{\operatorname{frac}\left\{9^{\wedge}\{3 / 2\}\right\}\left\{16^{\wedge}\{3 / 2\}\right\}\right.
$$

Figure 1. Student code example and how it renders in Moodle

It is considerably easier to read and comprehend this LaTeX code than if all the fractions had been expressed in their proper long form yet no information is lost. In this case there was a third fraction involved and the first student simply added this in words by typing "1 over" as shown in Fig. 2.

$$
1 \text { over } \frac{9^{3 / 2}}{16^{3 / 2}}
$$

Figure 2. Different idioms and non-standard forms in an expression

This is neither a formal nor a rigorous way of mathematical discussion. However in this case it was enough for the students to communicate and for peertutoring to occur (as it might for example in a non-formal face-to-face setting such as study group). Figure 3 shows how the first student asked the question (about a rule of fractions) and how the second student responded. (Very limited level of prior attainment in mathematics is required for this course and student peer tutoring is hence very important in helping students with basics that are not formally covered in the syllabus.) From the exchange in Figure 3 it is clear the respondent knew exactly what the first student was asking (with the three level nested fraction using mixed and ad-hoc conventions) and could reply with an answer. As with SMS text messaging, students are happy to use whatever form is quickest to type whilst still being comprehensible. Use of asterisk and forward slash $(* /)$ as multiplication and division operators was mentioned above. These are the symbols used on calculators, and are commonly used in computing. Students also used the caret character $\left(^{\wedge}\right)$ - which is not so common in computing and which they mostly likely learned from LaTeX - to denote superscript text e.g. $x^{\wedge} 2$ to say $\mathrm{x}$ squared. Sometimes they did not bother, or forgot, to type the command that marked this as LaTeX. Hence these fragments of raw code appeared in discussion postings. Although there may be downsides to this nonrigorous approach to notation, it points to the fact that these students find it useful for non-formal learning modes. Figure 4 shows (a rarer) example of using LaTeX more properly to format problems and interesting use of colouring.
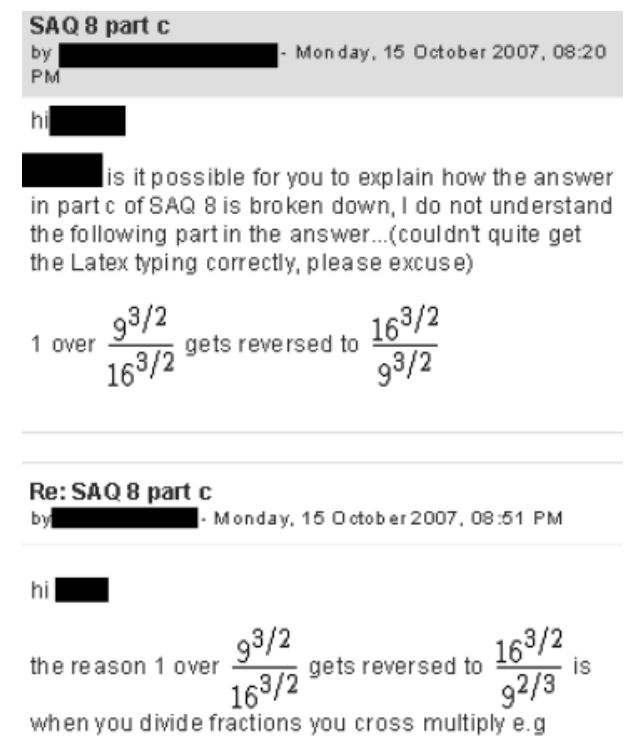

when you divide fractions you cross multiply e.g

$$
\begin{aligned}
& 1 \div \frac{9^{3 / 2}}{16^{3 / 2}}=\frac{1}{1} \div \frac{9^{3 / 2}}{16^{3 / 2}}=(\text { bottom line becomes the } \\
& \text { to } p \text { the top line becomes the bottom) } \frac{16^{3 / 2}}{9^{2 / 3}}
\end{aligned}
$$

have a look at the perp maths its good ifyou havent been at maths for a while, have gone through it myself and always have it open when i start to study it explains things a lot better.

\section{Figure 3. Online student discussion}

\section{Re: 2007 Q.3b \\ by Monday, 11 May 2009, 08:54 AM}

Yes, you are correct. Basically, the solution for each variable $x, y, z$ is a $3 \times 3$ determinant divided by another $3 \times 3$ determinant. The determinant on the bottom is the same for $x, y$ and $z$ - it is the $3 \times 3$ determinant formed by the coefficients of $x, y, z$. The determinant on the top is got by replacing a column by the right hand side constants. Thus, if

$a x+b y+c z=j$,

$d x+e y+f z=k$,

$g x+h y+i z=l$,

$\left[\begin{array}{lll}a & b & c \\ d & e & f \\ g & h & i\end{array}\right]\left[\begin{array}{l}x \\ y \\ z\end{array}\right]=\left[\begin{array}{l}j \\ k \\ l\end{array}\right]$

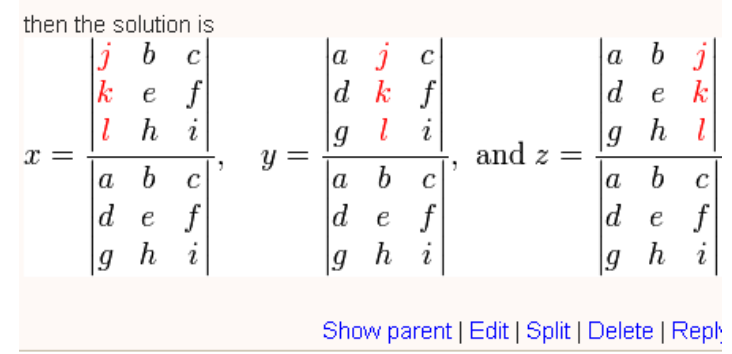

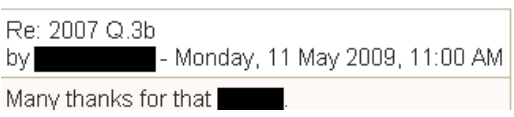

Figure 4. Discussion on Matrices 


\section{CONCLUSIONS AND FURTHER WORK}

Studies such as those of [7] and [8] both emphasise synchronous online communication as desirable. However there are many reasons, including technical ones such as scalability, for which the asynchronous mode is advantageous. Email and the web itself are mainly built on asynchronous slower forms of communication testifying to its value. The reflective aspect of asynchronous learning modes is well documented, though research in reflection in mathematics is hitherto mostly concentrated around student journals [14] [15] [16]. Here LaTeX is posited as a useful tool for facilitating asynchronous, online, collaborative learning of mathematics. There is much wider research on reflective and collaborative learning which may be of relevance to mathematics which is not explored here [5] [4]. The findings presented here are significant in that they are not consistent with some previous assumptions about LaTeX's suitability as a tool for online educational communication. It is found, for instance, to be easier to use than is often assumed. It allows for a "reusable, re-editable equation in a discussion thread", a functionality which [7] found to be lacking in many tools and which others have argued as part of any idealized mathematics learning framework [8]. Crucially it is found to be a pragmatic solution with a short learning curve. Some ability to code is a learning outcome of the specific programme which these students were part of. However SMS texting, instant messaging, email, social networking websites, discussion forums and other textbased mediums where syntax quickly evolves to forms that condense information are increasingly familiar to all students. The difficulty of coding should not be overestimated and its use should be considered in new contexts.

\section{REFERENCES}

[1] S. Fox, "Reflections of the benefits of the use of the new learning technologies in higher distance education through the prism of a case study," 11th International Conference on Open and Distance Learning, Cambridge, 2005.

[2] S. Fox, and K. MacKeogh, "Can elearning promote higher-order learning without tutor overload?," In Open Learning, vol. 18, Num 2, 2003, pp. 121-134 (doi:10.1080/02680510307410)

[3] S. Fox, and E. Walsh, "Task Orientated Online Learning (TOOL) - social interaction in an online learning environment," In Case Studies in Assessment of Student Learning in Higher Education. AISHE G. O' Neill, S. Huntley-Moore, and P. Race, Eds. Dublin: 2007, pp. 43-49.

[4] P. Dillenbourg, and D. Schnieder, "Collaborative learning and the Internet," http://tecfa.unige.ch/tecfa/research/CMC/colla/iccai95 1.html, 1995.
[5] D. W. Johnson, and R. T. Johnson, Cooperation and the Use of Technology. In Handbook of Research for Educational Communications and Technology. D. H. Jonassen, Ed,. New York: Simon and Schuster Macmillan, 1996, pp. 1017-1044.

[6] E., Costello, and S. Fox, "Use of online resources to aid student retention on first year mathematics and electronics courses: A preliminary report," EdTech 2006 - The Seventh Annual Irish Educational Technology Users' Conference, Sligo, 2006.

[7] L. Leventhall, "Bridging the Gap between face to face and online maths tutoring," International Congress of Mathematics Education 10, Copenhagen, Denmark, 2004.

[8] G. G. Smith, and D. Ferguson, "Diagrams and math notation in elearning: Growing pains of a new generation". In International Journal of Mathematical Education in Science and Technology, vol. 35 n5, Taylor and Francis, 2004, pp. 681-695.

[9] W3C.org, Mathematical Markup Language (MathML) Version 2.0 (Second Addition) W3C Recommendation 21 October 2003. http://www.w3.org/TR/MathML2/overview.html

[10] B. Loch, and C. McDonalds, "Synchronous chat and electronic Ink for distance sup-port in mathematics," In Journal of Online Education, J. L. Morrison Eds. February/March 2007, vol. 3, Issue 3

[11] J. Grackin, D. Ferguson, R. Izubuchi, and G. Smith, "Math and distance learning threaded discussions," In Proceedings of World Conference on Educational Multimedia, Hypermedia and Telecommunications. Chesapeake, VA: 2002, pp. 1819-1824

[12] D. McArthur, M., Lewis, and M, Bishay, The Roles of Artificial Intelligence in Education: Current Progress and Future Prospects. National Science Foundation (U.S.): Rand, 1993

[13] R., Remedios, and D. Lieberman, "I liked your course because you taught me well: The influence of grades, workload, expectations and goals on students' evaluations of teaching," In British Educational Research Journal. vol. 34, Issue 1, February 2008, pp. $91-115$ (doi:10.1080/01411920701492043)

[14] J. Moon, A Handbook of Reflective Practice and Experiential Learning. London: Routledge Falmer, 2004.

[15] D. Curry, "Journey into journal jottings: Mathematics as communication," In G. Iddo Ed. Adult Numeracy Development Theory, Research, Practice. New Jersey: Hampton Press, 2002.

[16] M. Lanigan, "Where are the examinations? Using learning Journals in mathematics assessment," In Case Studies in Assessment of Student Learning in Higher Education. G. O'Neill, S. Huntley-Moore, and P. Race Eds. Dublin: AISHE, 2007.

\section{AUTHORS}

E. Costello Oscail, Distance Education Centre, Dublin City University. eamon.costello@dcu.ie

S. Fox Oscail, Distance Education Centre, Dublin City University. foxs@dcu.ie

E. Walsh Oscail, Distance Education Centre, Dublin City University. elaine.walshe@dcu.ie

Submitted 10 June 2009. Published as resubmitted by the authors on 18 November 2009. 